\title{
T cells step up after SARS-CoV-2 vaccination with $B$ cell depletion
}

\author{
Thomas Berger $₫$ and Barbara Kornek
}

Detailed immunological analysis in a new study provides insight into the mechanisms of immune responses after SARS-CoV-2 vaccination in people who are receiving $B$ cell-depleting therapy for multiple sclerosis. The findings have implications for clinical practice, but more questions about SARS-CoV-2 vaccination and immunosuppression remain.

Refers to Apostolidis, S. A. et al. Cellular and humoral immune responses following SARS-CoV- 2 mRNA vaccination in patients with multiple sclerosis on anti-CD20 therapy. Nat Med. https://doi.org/10.1038/s41591-021-01507-2 (2021).

Patients with multiple sclerosis (MS) who are receiving $\mathrm{CD} 20$ monoclonal antibody (anti-CD20) therapy are at risk of severe COVID-19 (REF. ${ }^{1}$ ). The ability of the immune system to generate appropriate antibody responses to the SARS-CoV-2 virus or vaccine is reduced by such $B$ cell-depleting therapy ${ }^{2}$, and the extent of protection that the SARS-CoV-2 vaccine provides to individuals with low or absent humoral responses after vaccination has not yet been determined. However, $\mathrm{T}$ cell-mediated responses have been seen in patients receiving anti-CD20 therapy who are recovering from COVID-19 $\left(\mathrm{REF}^{3}\right)^{3}$, and some studies suggest that $\mathrm{T}$ cell responses contribute to the efficacy of SARS-CoV-2 vaccines in healthy individuals $^{4}$ and can protect against variants that evade neutralizing antibodies ${ }^{5}$. In combination, these findings suggest that $\mathrm{T}$ cell responses could be important for the efficacy of SARS-CoV-2 vaccination in people who are receiving $\mathrm{B}$ cell-depleting therapy. In a new study published in Nature Medicine, Apostolidis et al. ${ }^{6}$ investigated the mechanisms of humoral and cellular immune responses to SARS-CoV-2 mRNA vaccination, shedding light on the effects of the vaccine in the context of anti-CD20 therapy and the clinical implications.

The study by Apostolidis et al. ${ }^{6}$ involved 20 patients who were receiving anti-CD20 monotherapy for MS and 10 healthy control individuals. Analysis of humoral immune responses showed that patients receiving anti-CD20 therapy produced lower, qualitatively insufficient levels of antibodies against the SARS-CoV-2 spike protein or the receptor-binding domain as compared to healthy, untreated individuals. The attenuated antibody production was more obvious with a shorter duration since the last dose of anti-CD20 treatment. This observation led to the conclusion that vaccine-specific antibody responses depend on the interval between treatment dosing and vaccine administration. Further characterization of $\mathrm{B}$ cell responses in patients receiving anti-CD20 therapy showed that the levels of spike-protein-specific memory B cells were substantially lower in most patients than in healthy control participants.

The observed effects of anti-CD20 therapy on the humoral immune response were not unexpected and are in agreement with previous findings ${ }^{7,8}$. However, Apostolidis et al. provided novel insight into the immune response to SARS-CoV-2 mRNA vaccination through their commendably detailed and methodologically sophisticated analysis of the $\mathrm{T}$ cell responses in the context of anti-CD20 therapy. Extensive analyses of the dynamics of $\mathrm{B}$ cell and $\mathrm{T}$ cell subsets were performed to gain insights into the functional and defensive capabilities of these cells, revealing previously unknown interplay between B cells, antibodies and T cells after SARS-CoV-2 mRNA vaccination.

In all patients with MS receiving antiCD20 therapy, as well as in healthy individuals, SARS-CoV-2 vaccination induced strong, antigen-specific $\mathrm{CD} 4^{+} \mathrm{T}$ cell and $\mathrm{CD}^{+} \mathrm{T}$ cell responses. SARS-CoV-2-specific $\mathrm{T}$ cells were also identified in most patients receiving anti-CD20 treatment in another, subsequently published study ${ }^{7}$. However, the detailed analysis by Apostolides et al. ${ }^{6}$ demonstrated that the $\mathrm{T}$ cell response in patients receiving anti-CD20 therapy differed from that in healthy controls - CD4 ${ }^{+}$subsets were skewed, with a deficit in circulating follicular helper $\mathrm{T}$ cells that normally support antigen-specific $\mathrm{B}$ cell responses, and $\mathrm{CD}^{+}$ $T$ cell responses were strengthened, especially after the second vaccine dose.

\section{SARS-CoV-2 vaccination} induced strong, antigen-specific $\mathrm{CD}^{+}{ }^{+} \mathrm{T}$ cell and $\mathrm{CD} 8{ }^{+} \mathrm{T}$ cell responses

Of particular interest, Apostolidis et al. also identified differences in the $\mathrm{T}$ cell response among patients receiving anti-CD20 therapy. The pattern of the $\mathrm{T}$ cell response was closely related to the presence or absence of spike-protein-specific antibodies, which was in turn closely related to the presence or absence of circulating B cells. In patients who generated antibodies, $\mathrm{T}$ cell responses were less skewed. Although patients who did not generate a humoral vaccine response had selective deficits in circulating follicular helper T cells, their antigen-specific $\mathrm{CD}^{+} \mathrm{T}$ cell response was enhanced - not only were $\mathrm{CD}^{+} \mathrm{T}$ cell responses greater in magnitude in these patients, but they were also characterized by functional and sustained antiviral responses, indicated by their expression of pro-inflammatory cytokines and effector molecules, that were not seen in healthy control individuals.

The findings indicate that, despite poor antibody generation, SARS-CoV-2 mRNA vaccination can generate immune responses in individuals receiving anti-CD20 monotherapy that are likely to protect them against infection. Indeed, one study has indicated that $\mathrm{CD}^{+} \mathrm{T}$ cell responses improve survival of COVID-19 among patients with haematological cancer who are receiving anti-CD20 therapy ${ }^{9}$. The findings also provide important mechanistic insights into immune responses after vaccination. However, the complexity of $\mathrm{B}$ cell and $\mathrm{T}$ cell interactions in the context of vaccination, particularly in patients with altered immune responses owing to the presumed autoimmune nature of MS, is still far from being fully understood.

We also need to better understand the priming of specific $\mathrm{T}$ cell responses after depletion of $\mathrm{CD} 20^{+} \mathrm{B}$ cells and T cells in other scenarios, as adaptive immune responses might be influenced by various factors, such as the extent of B cell depletion, effects of immunosenescence, use of vaccines other than mRNA vaccines, and use of other immunosuppressive 
or immunomodulatory treatments. As an example, sphingosine-1-phosphate receptor modulators (S1PMs) are selective immunosuppressants that target $\mathrm{T}$ cell egression from lymph nodes, and patients with MS who are receiving an S1PM also have impaired antibody responses after SARS-CoV-2 mRNA vaccination $^{8}$. However, this impairment seems to depend on whether SARS-CoV-2 mRNA or vector-based vaccines are used ${ }^{10}$, suggesting that immune responses to the mRNA vaccine are impaired by the interaction of lipophilic S1PM molecules with mRNA nanoparticles.

C

responses to SARS-CoV-2

mRNA vaccination ... depend on the time since the last anti-CD20 treatment dose

In this context, the full clinical implications of the findings from Apostolidis et al. ${ }^{6}$ remain to be determined. However, one conclusion seems clear and has more general implications for the use of anti-CD20 treatments in MS and other neuroimmunological disorders: humoral or cellular responses to SARS-CoV-2 mRNA vaccination in patients with MS who are receiving anti-CD20 therapy strongly depend on the time since the last anti-CD20 treatment dose. However, on the basis of the seminal immunological analyses in this study, it seems likely that the immune response after vaccination depends on the extent of $B$ cell repopulation rather than the dosing intervals. Given that this repopulation varies between patients, we should routinely monitor B cells qualitatively and quantitatively in patients who are receiving anti-CD20 therapy and adjust treatment intervals individually according to patients' immune status. In the context of SARS-CoV-2 (and probably any other) vaccination, monitoring of $\mathrm{B}$ cells is also important to identify the best time point for vaccination of anti-CD20-treated patients to ensure maximal immune responses.

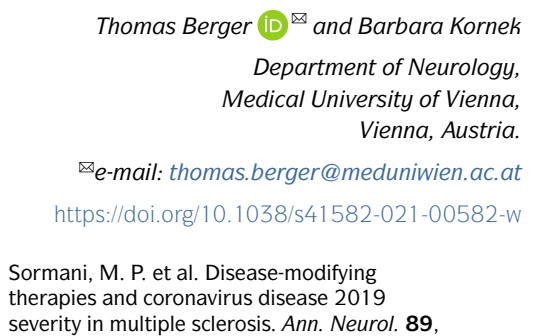
therapies and coronavirus disease 2019 severity in multiple sclerosis. Ann. Neurol. 89

1. Sormani, M. P. et al. Disease-modifying 780-789 (2021).
2. Sormani, M. P. et al. Effect of SARS-CoV-2 mRNA vaccination in MS patients treated with disease modifying therapies. EBioMedicine https://doi.org/ 10.1016/j.ebiom.2021.103581 (2021).

3. Iannetta, M. et al. T-cell responses to SARS-CoV-2 in multiple sclerosis patients treated with ocrelizumab healed from COVID-19 with absent or low anti-spike antibody titers. Mult. Scler. Rel. Dis. 55, 103157 (2021).

4. Painter, M. M. et al. Rapid induction of antigenspecific $\mathrm{CD}_{4}^{+}$cells guide coordinated humoral and cellular immune responses to SARS-CoV-2 mRNA vaccination. Immunity 54, 2133-2142 (2021).

5. Geers, D. et al. SARS-CoV-2 variants of concern partially escape humoral but not T-cell responses in COVD-19 convalescent donors and vaccinees. Sci. Immunol. 6, eabj1750 (2021).

6. Apostolidis, S. A. et al. Cellular and humoral immune responses following SARS-CoV-2 mRNA vaccination in patients with multiple sclerosis on anti-CD20 therapy. Nat Med. https://doi.org/10.1038/ s41591-021-01507-2 (2021).

7. Brill, L. et al. Humoral and T-cell response to SARSCoV-2 vaccination in patients with multiple sclerosis treated with ocrelizumab. JAMA Neurol. https:// doi.org/10.1001/jamaneurol.2021.3599 (2021).

8. Achiron, A et al. Humoral immune response to COVID-19 mRNA vaccine in patients with multiple sclerosis treated with high-efficacy disease-modifying therapies. Ther. Adv. Neurol. Disord. 14, https://doi. org/10.1177/17562864211012835 (2021).

9. Bange, E. M. et al. CD8 ${ }^{+} T$ cells contribute to survival in patients with COVID-19 and hematologic cancer. Nat. Med. 27, 1280-1289 (2021).

10. Rommer, P. S., Bsteh, G., Berger, T. \& Zettl, U. K. SARS-CoV-2 antibodies in multiple sclerosis patients depending on the vaccine mode of action? Mult. Scler. J. https://doi.org/10.1177/13524585211039128 (2021).

Competing interests

The authors declare no competing interests. 